\title{
TITLE:
}

\section{Decay process accelerated by tunneling in its very early stage}

$\operatorname{AUTHOR}(\mathrm{S}):$

Koide, T; Toyama, FM

\section{CITATION:}

Koide, T ...[et al]. Decay process accelerated by tunneling in its very early stage. PHYSICAL REVIEW A 2002, 66(6): 064102.

ISSUE DATE:

2002-12

URL:

http://hdl.handle.net/2433/50465

RIGHT:

Copyright 2002 American Physical Society 
PHYSICAL REVIEW A 66, 064102 (2002)

\title{
Decay process accelerated by tunneling in its very early stage
}

\author{
T. Koide ${ }^{1}$ and F. M. Toyama ${ }^{2}$ \\ ${ }^{1}$ Yukawa Institute for Theoretical Physics, Kyoto University, Kyoto 606-8502, Japan \\ ${ }^{2}$ Department of Communication and Information Sciences, Kyoto Sangyo University, Kyoto 603-8555, Japan
}

(Received 6 August 2002; published 9 December 2002)

\begin{abstract}
We examine a fast decay process that arises in the transition period between the Gaussian and exponential decay processes in quantum decay systems. It is usually expected that the decay is decelerated by a confinement potential barrier. However, we find a case where the decay in the transition period is accelerated by tunneling through a confinement potential barrier. We show that the acceleration gives rise to an appreciable effect on the time evolution of the nonescape probability of the decay system.
\end{abstract}

DOI: 10.1103/PhysRevA.66.064102

PACS number(s): 03.65.Xp

Quantum decay processes such as the nuclear alpha decay are usually well described by means of the exponential decay law; see, for example, Refs. [1-3]. Theoretically, however, deviations from the exponential law are expected in the beginning and also toward the end of a decay process [4]. It is understood that the quantum decay process in general proceeds through three different stages; initial, intermediate, and final. The initial stage is characterized by the Gaussian law, the intermediate stage by the exponential law, and the final stage by the power law. The decay speeds in the initial and final stages are smaller than that in the intermediate stage. In particular, the slow decay process in the Gaussian period leads to the possibility of the quantum Zeno effect [4], in which the decay process is decelerated by repeated measurements.

Many years ago, in his one-dimensional model analysis of a decaying system, Winter found that the speed of the decay process exhibits an irregular behavior in the transition period between the Gaussian and exponential periods [5]. Very recently Dicus et al. reexamined the same system, which consists of a particle which is initially confined in a region and leaks out by tunneling through a $\delta$-function potential barrier [6]. In the irregular decay process, the decay speed can be larger than that in the exponential region. Such a fast decay in the transition period is interesting in the sense that it may give rise to acceleration of the decay process by repeated measurements, which is the so-called anti- (inverse-) Zeno effect $[7,8]$.

The purpose of this report is to investigate the details of the fast decay process in the transition period between the Gaussian and exponential periods [9]. In particular, we explore how the decay process in the transition period depends on the strength of the confinement potential barrier with a finite potential width. It is usually expected that the decay is suppressed as the confinement becomes stronger because the potential barrier suppresses the time evolution of the wave function. However, we will illustrate a case where the decay in the transition period is accelerated by tunneling through a confinement potential barrier. Further, we show that the acceleration gives rise to an appreciable effect on the time evolution of the nonescape probability of the decay system.

We consider a model in one dimension with a potential that consists of an infinite repulsive wall at $x=0$ and a repulsive square barrier at $1<x<1+w$,

$$
V(x)=\left\{\begin{array}{cc}
0 & \text { for } 0<x<1 \\
h & \text { for } 1<x<1+w \\
0 & \text { for } 1+w<x,
\end{array}\right.
$$

where $h$ and $w$ are the height and width of the potential, respectively.

We assume that a particle of mass $m$ is initially confined within the potential barrier and it leaks out in time. The time evolution of the system is determined by the time-dependent Schrödinger equation

$$
i \frac{\partial \phi(x, t)}{\partial t}=H \phi(x, t), \quad H=-\frac{\partial^{2}}{\partial x^{2}}+V(x),
$$

where the units are such that $\hbar=1$ and $2 m=1$. We solve Eq. (2) numerically using the implicit solution method [10], with the unitary time-evolution operator $U \equiv(1-i H \delta t / 2) /(1$ $+i H \delta t / 2)$, where $\delta t$ is the time mesh. For the range of $x$, we take $[0,500]$. In order to suppress reflection of the wave function at the boundary $(x=500)$ of the model space, we assume an imaginary potential at $x \geqslant 490$.

For the initial wave function of the particle which is confined in the region of $0 \leqslant x \leqslant 1$, we assume

$$
\phi(x, 0)=\sqrt{2} \sin \pi x .
$$

It is understood that $\phi(x, 0)=0$ for $x>1$. The wave function leaks out in time by tunneling through the potential barrier $V(x)$. The energy expectation value $\langle H\rangle$ at $t=0$ is $\pi^{2}$. In numerical illustrations we choose the height of the potential barrier such that $h>\pi^{2}$. In this sense we regard the process as a tunneling decay process.

We define probability $P(a, t)$ for the particle being in the interval $0 \leqslant x \leqslant a$ at $t$ by

$$
P(a, t)=\int_{0}^{a} d x|\phi(x, t)|^{2}
$$

If we set $a=1+w, P(1+w, t)$ represents the probability that the particle is confined by the potential barrier. For $w$ we consider a few different values. In order to compare the results for different values of $w$, we set $a$ such that $a>1$ $+w_{\text {max }}$. Throughout this paper, we take $a=4$. The $P(a, t)$ is 


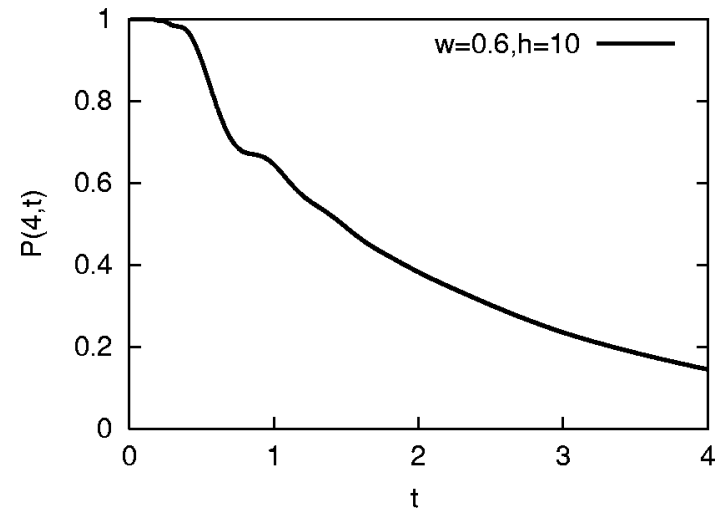

FIG. 1 . The time evolution of the nonescape probability $P(4, t)$ for $w=0.6$ and $h=10$. The units are such that $\hbar=1$ and $2 m=1$.

a kind of the nonescape probability, which is the probability that the particle has not escaped from the potential by time $t$ $[11,12]$.

Next we introduce function $g(a, t)$ defined by

$$
g(a, t)=\frac{d P(a, t) / d t}{P(a, t)} .
$$

If probability $P(a, t)$ decays exponentially, that is, if $P(a, t) \propto e^{-\gamma t}$, then $g(a, t)$ is independent of time,

$$
g(a, t)=-\gamma
$$

If $P(a, t)$ obeys the Gaussian decay law, that is, if $P$ $\propto e^{-t^{2} / \tau}, g(a, t)$ is proportional to $t$,

$$
g(a, t)=-2 t / \tau
$$

Thus, from the $t$ dependence of $g(a, t)$ we can see how well $P(a, t)$ obeys the exponential law or the Gaussian law.

Figure 1 shows the nonescape probability $P(a, t)$, where the potential height and width are taken as $h=10$ and $w$ $=0.6$, respectively. In the initial period $0 \leqslant t \leqslant 0.3$, the decay is extremely slow. The period should correspond to the Gaussian decay process. In the period of $t \gtrsim 2$, the system is subject to the exponential decay law. Between the two stages, there is a period $(0.3 \lesssim t \lesssim 2)$ in which the decay process is neither Gaussian nor exponential. This is the transition period. In the period the decay of the nonescape probability seems to be much faster than that in the exponential period.

The function $g(4, t)$ calculated from $P(4, t)$ of Fig. 1 is shown by the solid line in Fig. 2. In the period of $t \gtrsim 2$, $g(4, t)$ is almost constant, which means that the decay process is exponential. In the period of $0 \leqslant t \leqslant 0.3, g(4, t)$ is not exactly proportional to $t$, i.e., the decay process in the initial stage slightly deviates from the Gaussian decay law. However, the decay speed in this period is still smaller than that in the exponential period. Corresponding to the rapid decay process seen in $P(t)$ of Fig. 1, the maximum of $|g(4, t)|$ is obtained in the transition period $(t \sim 0.6)$. The $g(4, t)$ starts from zero at $t=0$. Thus, the quantum Zeno effect is possible when we repeat measurements with a sufficiently small time

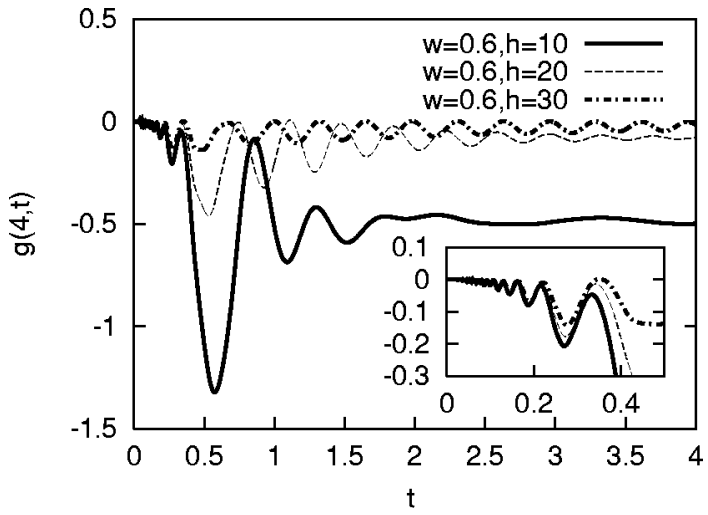

FIG. 2. The time evolution of $g(4, t)$ for a fixed width $w=0.6$. The solid, dashed, and dot-dashed lines show $g(4, t)$ for the heights $h=10,20$, and 30 , respectively. The units are such that $\hbar=1$ and $2 m=1$.

interval. On the other hand, the anti-Zeno effect is possible by repeated measurements only when the net decay rate of the fast decay process in the transition period is large compared to that of the slow decay process in the Gaussian period (see, footnote Ref. [13]).

In order to see how the decay process in the transition period depends on the strength of the potential barrier, we examine $g(4, t)$ for various potential heights and widths. The dashed and dot-dashed lines in Fig. 2 show the $g(4, t)$ for $h=20$ and $h=30$, respectively with a fixed width $w=0.6$. The decay process in the Gaussian period $0 \leqslant t \leqslant 0.3$ does not depend strongly on the potential height. This is because in the initial stage the higher-energy components of the initial wave function relative to the potential height contribute mainly to the decay of the system. On the other hand, the decay speed in the exponential period becomes much smaller as the confinement becomes stronger. The fast decay in the transition period depends strongly on the potential height. It tends to be suppressed as the confinement becomes stronger.

In Fig. 3, we show $g(4, t)$ for various potential widths and a fixed height $h=15$. The decay process in the Gaussian period $0 \leqslant t \leqq 0.3$ does not seem to depend strongly on the

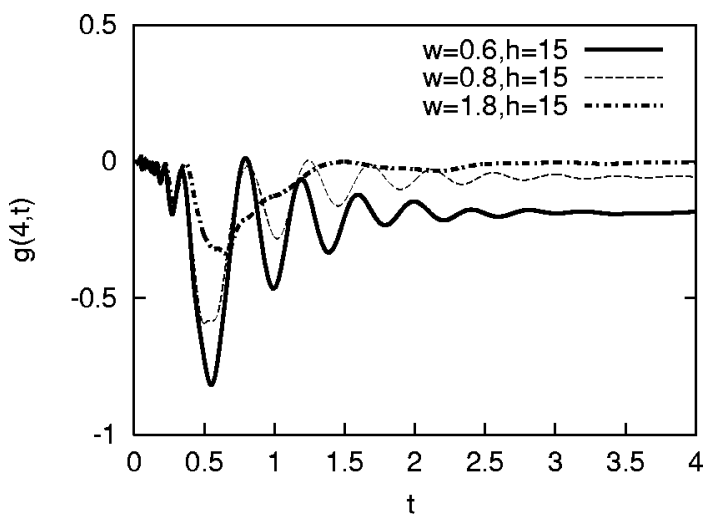

FIG. 3. The time evolution of $g(4, t)$ with a fixed height $h$ $=15$. The solid, dashed and dot-dashed lines are the $g(4, t)$ for $w$ $=0.6,0.8$, and 1.8, respectively. The units are such that $\hbar=1$ and $2 m=1$. 


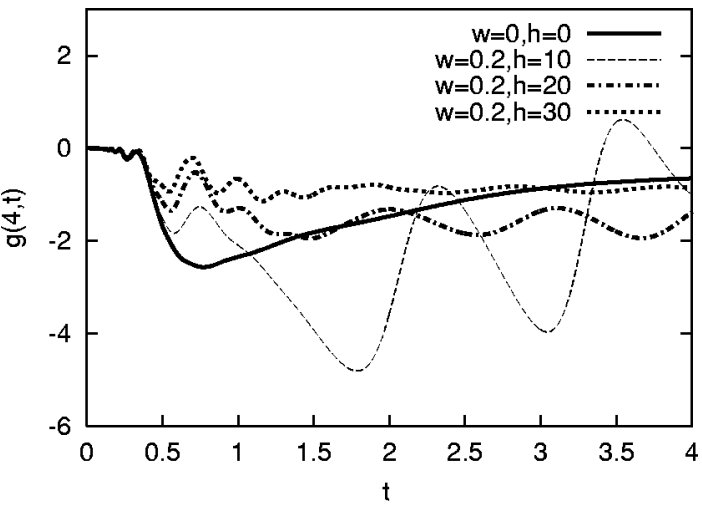

FIG. 4. The time evolution of $g(4, t)$ for a fixed width $w=0.2$. The dashed, dot-dashed, and dotted lines are the $g(4, t)$ for the widths $h=10,20$, and 30, respectively. The solid line shows the $g(4, t)$ for no potential barrier. The units are such that $\hbar=1$ and $2 m=1$.

potential width. On the other hand, the decay rate of the exponential period becomes much smaller as the potential width becomes broader. This is due to the increase of the confinement strength. The decay speed in the transition period becomes larger as the potential width becomes narrower.

As we have shown, the speed of the fast decay process becomes smaller as the potential barrier becomes stronger. Thus, we might guess that the fastest decay will be obtained in the decay process with no potential barrier. However, as we will show in the following, potential barriers with appropriate widths and heights can accelerate the decay process.

In Fig. 4, the solid line shows $g(4, t)$ calculated with no potential barrier. The dashed, dot-dashed, and dotted lines exhibit $g(4, t)$ calculated with the potential barriers with $h$ $=10,20$, and 30 and fixed width $w=0.2$, respectively. It should be noted that $w=0.2$ is much thinner than those used in Figs. 2 and 3, and therefore, in this case, the confinement is very weak compared to that in Figs. 2 and 3. In the Gaussian period, the potential dependence of the decay speed is not very appreciable. However, in the transition region, the decay speed becomes faster as the potential height becomes lower. For $h=10$, the maximum decay speed at $t \sim 1.8$ exceeds that for no potential barrier at $t \sim 0.7$.

As shown in Fig. 5, such an acceleration of the decay speed by tunneling gives rise to an appreciable difference in the time evolution of $P(4, t)$. The solid and dashed lines are the $P(4, t)$ for no potential barrier and for the potential barrier with $w=0.2$ and $h=10$, respectively. The nonescape probability for $h=10$ becomes smaller than that for no potential barrier at $t \sim 1.5$. At this time the residual nonescape probability is still about $10 \%$. In this sense, the effect of this acceleration cannot be ignored. On the other hand, for $h$ $=20$, at the time region in which the nonescape probability becomes smaller than that for no potential barrier, the residual nonescape probability is negligibly small.

The fluctuations in the behaviors of $g(4, t)$ indicate that the decay processes are still in the transition period from the Gaussian to exponential period. However, as shown in Fig. 5, the decay process has been almost completed before $t=4$. Therefore, even if the decay process proceeds to the expo-

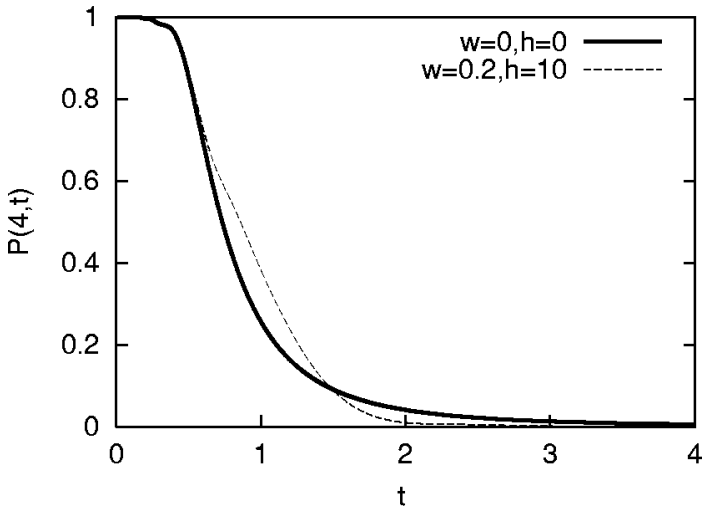

FIG. 5. The time evolution of the nonescape probabilities $P(4, t)$. The solid and dashed lines are the $P(4, t)$ for no potential barrier and for the potential barrier with $w=0.2$ and $h=10$, respectively. The units are such that $\hbar=1$ and $2 m=1$.

nential period eventually, the exponential decay has no importance in this case. The stability of quantum system is usually characterized by the magnitude of the imaginary part of the pole that gives the inverse of the lifetime in the exponential period [4-6]. The result that we have shown implies that such a pole analysis may not be effective for highly unstable systems [14].

In Fig. 4, we have seen that the acceleration by tunneling can be obtained for $h \lesssim 10$. Next, we investigate the acceleration with a fixed height. We examine the time evolution of $g(4, t)$ for $h=10$ [15]. The dashed, dot-dashed, and dotted lines in Fig. 6 exhibit $g(4, t)$ calculated for the potential barriers with $w=0.2,0.4$, and 0.6 and fixed height $h=10$, respectively. One can see that the decay rate becomes larger for thinner potential widths. For $w=0.2$, the maximum decay speed exceeds that for no potential barrier. Thus, one sees that the acceleration of the decay speed by tunneling can be obtained when the strength of the confinement by the potential barrier is sufficiently weak. In our illustrations, for $h$ $\lesssim 10$ and $w \leq 0.2$, the accelerations are remarkable.

Finally, we mention that the $g(4, t)$ represented by dotted line in Fig. $4(w=0.2$ and $h=10)$ takes positive values

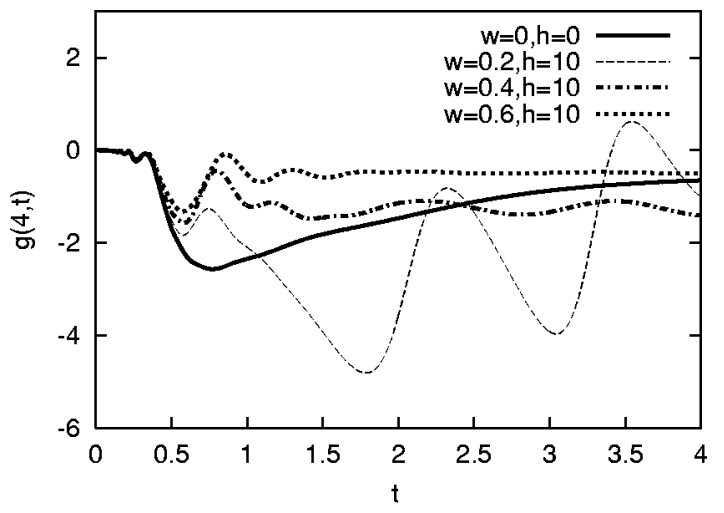

FIG. 6. The time evolution of $g(4, t)$ with a fixed height $h$ $=10$. The dashed, dot-dashed, and dotted lines are the $g(4, t)$ for the heights $w=0.2,0.4$, and 0.6 , respectively. The solid line shows the $g(4, t)$ for no potential barrier. The units are such that $\hbar=1$ and $2 m=1$. 
around $t \sim 3.5$. Recall that the probability current $j(a, t)$ is related to the nonescape probability $P(a, t)$ by $j(a, t)$ $=-d P(a, t) / d t=-g(a, t) P(a, t)$. This means that, if $g(a, t)$ is positive, $j(a, t)$ is negative. However, in Refs. $[5,16]$ the negative currents were obtained at very late time region after the exponential decay period. Our result implies that in a highly unstable quantum system the negative current can occur even at the initial stage after the Gaussian period.

We have investigated the fast decay process in the transition period between the Gaussian and exponential decay processes. In most cases of the tunneling process, the decay speed becomes smaller as the potential barrier becomes stronger. As a special case, we have found that the fast decay process can be remarkably accelerated by tunneling through potential barriers with appropriately small widths and heights. A detailed analysis of the acceleration of the fast decay process by tunneling is a future project.

We would like to thank Professor Y. Nogami for useful discussions and comments. This work was supported in part by the Ministry of Education, Culture, Science, Sports and Technology of Japan.
[1] G. Gamow, Z. Phys. 51, 204 (1928); 52, 510 (1928); E.U. Condon and R.W. Gurney, Nature (London) 112, 439 (1928); Phys. Rev. 33, 127 (1929).

[2] V. Weisskopf and E.P. Wigner, Z. Phys. 63, 54 (1930); 65, 18 (1930)

[3] G. Breit and E.P. Wigner, Phys. Rev. 49, 519 (1936).

[4] For review articles, see L. Fonda, G.C. Ghirardi, and A. Rimini, Rep. Prog. Phys. 41, 587 (1978); H. Nakazato, M. Namiki, and S. Pascazio, Int. J. Mod. Phys. B 10, 247 (1996).

[5] R.G. Winter, Phys. Rev. 123, 1503 (1961).

[6] D.A. Dicus, Wayne W. Repko, Roy F. Schwitters, and Todd M. Tinsley, Phys. Rev. A 65, 032116 (2002).

[7] W.C. Schieve, L.P. Horwitz, and J. Levitan, Phys. Lett. A 136, 264 (1989); A.G. Kofman and G. Kurizki, Nature (London) 405, 546 (2000); P. Facchi, H. Nakazato, and S. Pascazio, Phys. Rev. Lett. 86, 2699 (2001), and references therein.

[8] M.C. Fischer, B. Gutiérrez-Medina, and M.G. Raizen, Phys. Rev. Lett. 87, 040402 (2001).
[9] The transition region between the exponential and power periods has been examined; see W. van Dijk and Y. Nogami, Phys. Rev. C 65, 024608 (2002).

[10] A. Goldberg, H.M. Schey, and J. Schwartz, Am. J. Phys. 35, 177 (1967).

[11] R.B. Frey and E. Thiele, J. Chem. Phys. 48, 3240 (1968).

[12] G. García-Calderón, J.L. Mateos, and M. Moshinsky, Phys. Rev. Lett. 74, 337 (1995); Ann. Phys. (N.Y.) 249, 430 (1996).

[13] In order to observe the anti-Zeno effect, we have to repeat the measurements with a time interval $\tau$ that satisfies the condition $F(\tau)=\int_{0}^{\tau} d t\left[g(4, t)+\gamma_{\text {exp }}\right]<0$, where $\gamma_{\text {exp }}$ is a decay rate in the exponential region.

[14] A.G. Kofman, G. Kurizki, and B. Sherman, J. Mod. Opt. 41, 353 (1994).

[15] Notice that we consider the tunneling phenomena, so we do not investigate the lower height than $H=10$.

[16] Y. Nogami, F.M. Toyama, and W. van Dijk, Phys. Lett. A 270, 279 (2000). 